\title{
Lithotripsy of an impacted calcified stone in the cystic duct accompanied by cholecystitis in severe Crohn's disease
}

\author{
U Stölzel, C Koszka, M Gregor, K Ziegler, T Zimmer, E O Riecken
}

\begin{abstract}
A 35 year old women patient with Crohn's disease and previous multiple abdominal operations presented with a calcified stone of $12 \mathrm{~mm}$ diameter in the cystic duct giving rise to cholecystitis. The surgeons declined to operate because of extensive intra-abdominal adhesions caused by multiple intestinal resections and chronic enterocutaneous fistulas. It was possible to fragment the stone in three lithotripsy sessions. The fragments were excreted spontaneously through the ductus choledochus and the cholecystitis was cured by antibiotic treatment. The patient remained symptom free after 12 months.

(Gut 1993; 34: 1145-1147)
\end{abstract}

Biliary extracorporeal lithotripsy has been considered as one of the alternatives to surgery for the treatment of stones in the bile ducts and in the gall bladder in selected patients. ${ }^{1-4}$ Although fragmentation of slightly calcified stones in the gall bladder has been described, usually these patients were excluded from shock wave lithotripsy. ${ }^{5}$ Lithotripsy of a densely calcified gall bladder stone has been described in a case report. ${ }^{6}$ Obstruction of the cystic duct and cholecystitis are regarded as absolute contraindications for gall stone lithotripsy. We report for the first time the successful lithotripsy of a calcified cystic duct stone despite these relative and absolute contraindications in a patient with Crohn's disease. Furthermore, we followed up the course of the accompanying cholecystitis for 12 months and describe its complete cure.

\section{Case report}

At the time of presentation, the patient was 35 years old. Since 1981, she had suffered from Crohn's disease, which had had a complicated course. Multiple operations were necessary because of enterocutaneous, enteroenteral, and rectovaginal fistulas, a fistula perforation with faecal peritonitis, ileum and colon stenosis, and abscess formations of the abdominal wall. In total, $30 \mathrm{~cm}$ of ileum, $60 \mathrm{~cm}$ of jejunum, and the complete colon had been resected and an ileosigmoidostomy was performed. Crohn's disease was treated with $50 \mathrm{mg} / \mathrm{d}$ azathioprine and $8 \mathrm{mg} / \mathrm{d}$ methylprednisone. The chronic enterocutaneous fistula system was drained by a stoma bag. The patient had a five year history of cholecystolithiasis.

The reason for hospital admission was severe colicky pain in the right upper abdomen for two months. Intake of food by mouth was nearly

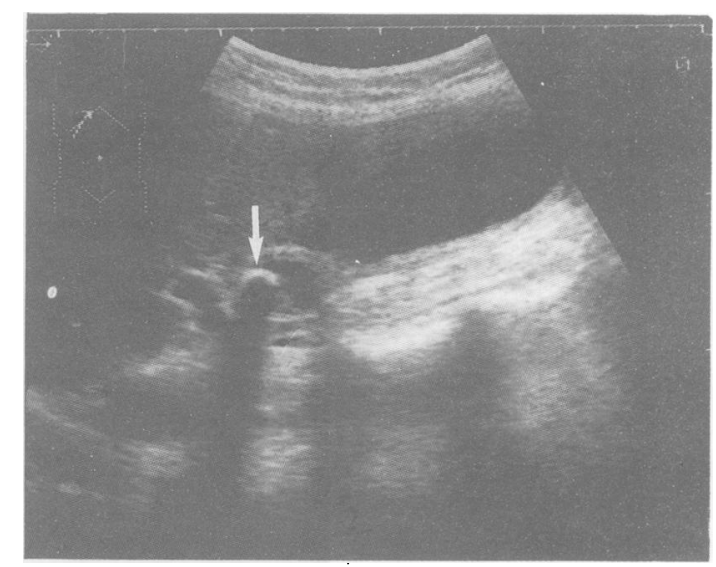

Figure 1: Cystic duct stone with acoustic shadowing shown by sonography in a patient with Crohn's disease.

impossible because of postprandial vomiting and pain. Furthermore, fever attacks with temperatures up to $39.5^{\circ} \mathrm{C}$ occurred. On admittance, the laboratory findings were as follows: white cell count $7 \cdot 9 \times 10^{9} / 1$, red blood cell count $3.4 \times 10^{12} / 1$, haemoglobin $103 \mathrm{~g} / \mathrm{l}$, platelet count $720 \times 10^{9} / 1$, alkaline phosphatase $342 \mathrm{U} / 1$, gammaGT $42 \mathrm{U} / 1$. Serum activities of aspartate transaminase alanine transaminase, amylase, lipase, the concentrations for total bilirubin, glucose, potassium, sodium, creatinin, and the prothrombin time were in the normal range. Sonography showed a thickened gall bladder wall with a halo of oedema around the gall bladder. In projection on to the cystic duct, a stone of $12 \mathrm{~mm}$ diameter with acoustic shadowing was seen (Fig 1). The abdominal $x$ ray film of the upper right abdominal quadrant showed a round calcification figure (Fig 2). The endoscope retrograde cholangiography confirmed the diagnosis of an occluding stone in the cystic duct (Fig 3).

A sphincterotomy was not performed. During her clinical treatment, the patient was fed artificially and repeatedly received $15 \mathrm{mg}$ doses of pentazocine intravenously for pain relief. Cefotiame $2 \mathrm{~g}$ intravenously two times daily was used for antibiotic treatment of the cholecystitis.

\section{Methods}

For lithotripsy, a combination lithotriptor MPL 9000 (Dornier) was used. The shock waves were produced by spark discharge, focused by a reflector, and transmitted by a coupled waterpillow. In total, three treatments with 1600 , 1650 , and 3010 shock waves with a mean voltage of $17 \mathrm{kV}$ were necessary.

The patient was treated in prone position 


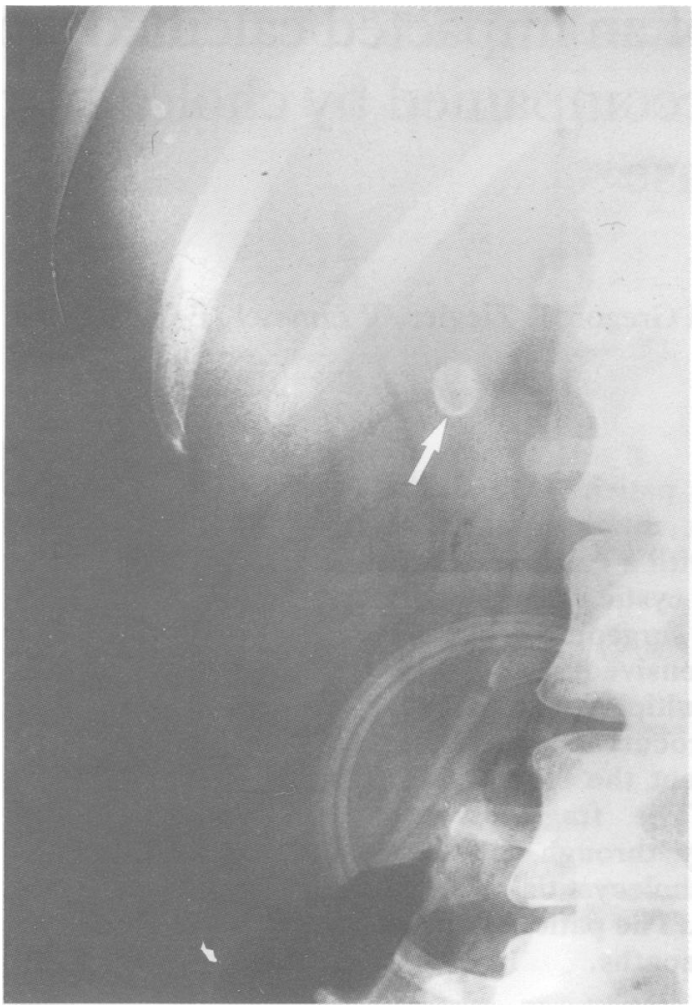

Figure 2: $\mathrm{x}$ Ray of the right upper abdomen shows the calcified stone and the stoma bag in a patient with Crohn's disease.

without narcosis. The stone was positioned in the shock wave focus under continous sonographic control. Before each session the patient received $40 \mathrm{mg}$ promethacine by mouth and $15 \mathrm{mg}$ piritramide intravenously for analgesia.

\section{Results}

After the second treatment we saw by sonography a splitting of acoustic shadowing into

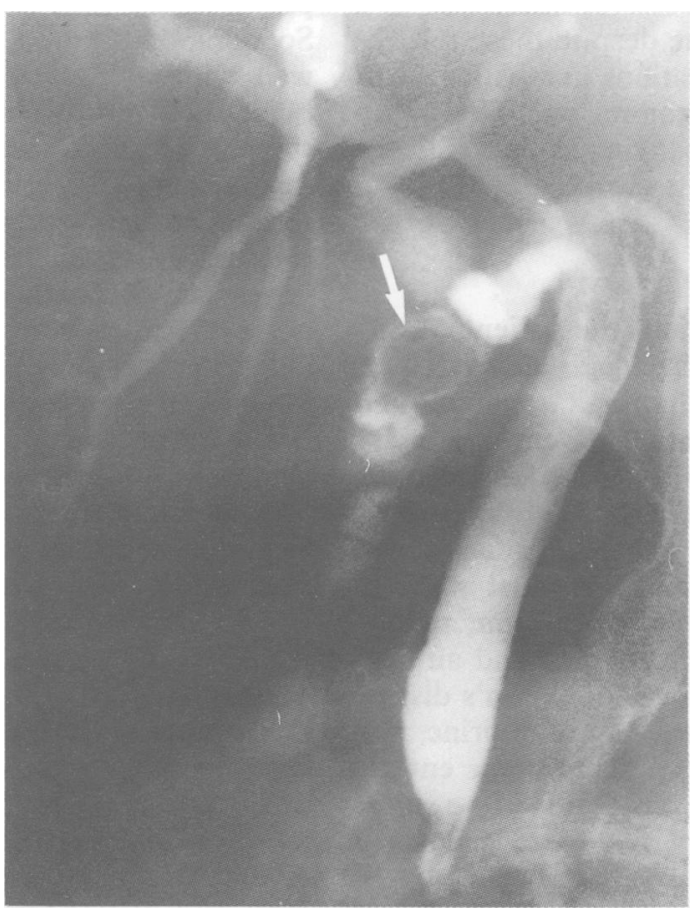

Figure 3: Endoscopic retrograde cholangiography shows an impacted stone in the cystic duct in a patient with Crohn's disease. three parts as a sign of fragmentation. On the day after the third treatment, the stone in the cystic duct was not detectable and the gall bladder had collapsed. The gall bladder wall was laminated and thickened. Three days after the last lithotripsy, the plain abdominal $x$ ray showed no calcifications in the right upper abdomen. From these findings and from the clinical course, we concluded that the fragments had passed spontaneously into the duodenum. After the third treatment, the colic episodes in the right upper abdomen stopped and food by mouth was started without problems. While the serum activities of alanine transaminase and aspartate transaminase remained normal during and after lithotripsy, the activities of the slightly raised alkaline phosphatase and gammaGT persisted.

Endoscopic retrograde cholangiography showed the cystic duct and the ductus choledochus to be free of stones. An oral cholecystogram was not performed after lithotripsy. The thickening of the gall bladder seen by sonography subsided after two weeks. Oral antibiotic treatment was continued while the patient was ambulant after admission for one month and ursodesoxycholic acid $750 \mathrm{mg} / \mathrm{d}$ was given permanently for the prevention of gall stone recurrence. So far we have continued to study the patient for 12 months. She had already gained $5 \mathrm{~kg}$ in weight four months after treatment. Monthly sonographic controls showed an inconspicuous gall bladder.

\section{Discussion}

The usual treatment for cholecystitis with occlusion of the cystic duct is surgical cholecystectomy. Because of several previous abdominal operations including colon resection and removal of a total of $90 \mathrm{~cm}$ of small intestine, extensive abdominal adhesions and a system of enterocutaneous fistulas secondary to severe Crohn's disease, the surgeons in our hospital refused to operate. Therefore, we carried out a shock wave lithotripsy of the impacted stone in the cystic duct despite contraindications. Endoscopic retrograde stone extraction was a possible alternative, although difficulties in cannulating the cystic duct and a high risk of sphincterotomy because of complicated Crohn's disease were to be expected. Furthermore, the endoscopic mobility was limited as a result of duodenal adhesions. Vermeersch et al have reported on a case of successful percutaneous endoscopic removal of an impacted stone in the cystic duct accompanied by cholecystitis.'

Although cholecystitis stopped in the three months follow up in that case, we did not consider this technique for our patient because of her history of fistulas. Obstruction of the cystic duct accompanied by cholecystitis is seen as an absolute contraindication and a calcified stone as a relative contraindication for lithotripsy. Our results show that even under these conditions a successful treatment of cholecystitis with stone obstruction of the cystic duct is possible without operation in carefully selected patients.

\footnotetext{
1 Sauerbruch T, Delius M, Paumgartner G, Holl J, Wess O, Weber W, et al. Fragmentation of gallstones by extracorporeal shock waves. $N$ Englf Med 1986; 318: 818-22.
} 
2 Sackmann M, Pauletzki J, Sauerbruch T, Holl J, Schelling G, Paumgartner G. The Munich gallbladder lithotripsy study. Results of the first 5 years with 711 patients. Ann Intern Med 1991; 114: 290-6.

3 Sauerbruch $\boldsymbol{M}$, Stern $\boldsymbol{M}$. Fragmentation of bile duct stones by extracorporeal shockwaves. Gastroenterology 1989; 96: 14652.

4 Staritz M, Rambow A, Grosse A, Hurst A, Floth A, Mildenberger $\mathrm{P}$, et al. Electromagnetically generated extra-
corporeal shockwaves for fragmentation of extra- and intracorporeal shockwaves for fragmentation of extra- and intra-
hepatic bile duct stones: indications, success and problems during a 15 month clinical experience. Gut 1990; 31: 222-5.

Sackmann M, Pauletzki J, Delius M, Holl J, Neubrand $M$, Sauerbruch $\mathrm{T}$, et al. Noninvasive therapy of gallbladder calculi with a radiopaque rim. Gastroenterology 1992; 102: 988-93.

6 Albert MB, Fromm H, Shehan CM. Densely calcified gallstone treated successfully with piezoelectric lithotripsy. Gastrotreated successfully with
enterology $1990 ; 98: 777-9$.

7 Vermeersch B, Steyaert P, Hoste P. Percutaneous endoscopic removal of an impacted cystic duct stone. Gastrointest Endosc 1991; 37: 583-4. 